\title{
Single-site, five-year experience with human eosinophil isolation by density gradient centrifugation and CD16 immunomagnetic negative separation
}

\author{
Yun Cao ${ }^{1}$, Sooncheon Shin ${ }^{1}$, Daniela J. Carroll', Jeremy A. O'Sullivan ${ }^{1}$ and Bruce S. Bochner ${ }^{1,2^{*}}$ (1)
}

\begin{abstract}
Objective: Little has been reported regarding the reliability of methods for the purification of human blood eosinophils. We retrospectively reviewed our experience with 350 consecutive eosinophil isolations.

Results: Between January 2014 and December 2018, we conducted 350 eosinophil purifications from 83 donors. Absolute eosinophil count (AEC), calculated from hospital complete blood counts when available $(n=289)$, ranged from 32 to 1352 eosinophils/ $\mu \mathrm{L}$ ( $\bar{x} \pm$ SD: $179 \pm 136 / \mu \mathrm{L}$ ). Eosinophil yields ranged from 0.4 to 24.4 million cells per $20 \mathrm{~mL}$ of blood drawn ( $\bar{x} \pm$ SD: $3.1 \pm 1.9$ million eosinophils) with > 98\% purity. Comparing AEC to actual yield, recovery was $87 \% \pm 29 \%(\bar{x} \pm$ SD) and AEC strongly correlated with yield. To explore the reproducibility of yield, a subsequent analysis was limited to those donors drawn $\geq 3$ times $(N=35)$, and there was no difference in the average coefficient of variation for yield between allergic and non-allergic donors. Viability of isolated eosinophils was consistently $>95 \%$ and after $24 \mathrm{~h}$ of culture did not differ between allergic and non-allergic donors. We conclude that this immunomagnetic separation method for human eosinophil isolation from whole blood is a reliable, reproducible technique for obtaining an average of $87 \%$ yield with high purity and viability.
\end{abstract}

Keywords: Human eosinophil purification, Blood, Methods, Purity, Yield, Immunomagnetic separation

\section{Introduction}

Human eosinophils, one of the less common types of leukocytes, are felt to contribute to homeostatic, immune and pathologic conditions [1]. Normal numbers of eosinophils in the blood are typically defined as $\leq 500 /$ $\mu \mathrm{L}$. Blood eosinophilia, defined as an absolute eosinophil count $>500 \mu \mathrm{L}$, and hypereosinophilia, defined as any absolute eosinophil count $\geq 1500 / \mu \mathrm{L}$, can be seen in a variety of conditions ranging from atopic, gastrointestinal and parasitic diseases to drug reactions, immune

${ }^{*}$ Correspondence: bruce.bochner@northwestern.edu

${ }^{2}$ Division of Allergy and Immunology, Northwestern University Feinberg School of Medicine, 240 E. Huron St., Room M-306, Chicago, IL 60611, USA Full list of author information is available at the end of the article deficiencies, malignancies and hematopoietic disorders $[2,3]$. Because of the potential for eosinophils to directly cause tissue damage, several therapies have been developed that selectively target eosinophils, and as a result, we now know that eosinophils directly contribute to disease pathophysiology in disorders ranging from asthma to eosinophilic granulomatosis with polyangiitis to hypereosinophilic syndromes [4-10].

Our knowledge of eosinophil biology has greatly benefited from the availability of methods that allow their purification for study in vitro. These methods initially involved intricate, multi-step density gradient centrifugation techniques, as eosinophils have the highest density of all leukocytes, overlapping in this regard only with neutrophils [11]. Major advances in the field occurred with 
the development of activation-based and antibody-based positive and negative selection methods, such as those that incorporate so-called immunomagnetic approaches [12-14], as well as the use of flow cytometric cell sorting [15]. However, little has been reported regarding their reproducibility and yield, as well as donor-to-donor variability and within-donor reproducibility across multiple donors and blood draws. Therefore, we have retrospectively reviewed our experience over a five-year period with 350 consecutive human eosinophil isolation preparations from whole blood using density gradient centrifugation, red blood cell (RBC) hypotonic lysis and immunomagnetic removal of contaminating neutrophils using CD16 antibody and herein describe key features of this methodologic approach.

\section{Main text \\ Methods \\ Human blood donors}

Written informed consent for blood donation was obtained using an institutional review board-approved protocol at the Northwestern University Feinberg School of Medicine. The allergic status of donors was determined based on their history (food allergy, atopic dermatitis, allergic rhinitis, and/or asthma) together with a history of needing medications (either actively at the time of the blood draw or on an as-needed basis) for one or more of these conditions. Donors were not allowed to give blood if they had received systemic corticosteroids in the prior 2 months or if they were receiving biologics for any of these conditions within the past year. For most blood donations, a separate vial of blood was drawn and provided to our hospital laboratory so that a complete blood count with differential (CBC) could be obtained. Information from the $\mathrm{CBC}$ results were used to determine starting blood eosinophil counts $\left(\right.$ cells $/ \mathrm{mm}^{3}$ ) for calculation of yield, as well as to screen for any hematologic abnormalities such as anemia.

\section{Eosinophil isolation}

Eosinophils were isolated from mildly allergic and nonallergic donors essentially as described [16]. In brief, $0.1 \mathrm{M}$ EDTA-anticoagulated blood ( $4 \mathrm{~mL}$ per $60 \mathrm{~mL}$ of blood) was diluted 1:3 with phosphate-buffered saline, then quantities of $40 \mathrm{~mL}$ of diluted blood were layered onto a $10 \mathrm{~mL}$ cushion of Percoll (Sigma-Aldrich, St. Louis, MO) adjusted to a specific density of $1.090 \mathrm{gm} / \mathrm{mL}$ in $50 \mathrm{~mL}$ conical tubes and centrifuged at $335 \mathrm{~g}$ for $20 \mathrm{~min}$ at room temperature without braking (Beckman Coulter Allegra model X-15R). Mononuclear cells, platelets and basophils in the upper layer were removed. Granulocytes and RBCs were collected from the pellets, and a 30-s RBC lysis with ice-cold water was performed three times consecutively. Finally, anti-human CD16 magnetic microbeads (Miltenyi Biotec, Auburn, CA) were added to label neutrophils and negatively select eosinophils over a magnetized mesh column. Purity was determined by cytocentrifugation (Shandon Inc., Pittsburgh, PA) and Kwik-Diff (Thermo Fisher Scientific, Waltham, MA) staining. Viability was determined by flow cytometry (Becton Dickenson LSR II) and 4'6-diamidino-2-phenylindole dihydrochloride (DAPI) exclusion (Thermo Scientific). Cells were cultured in RPMI 1640 medium containing $10 \%$ fetal calf serum and $0.5 \%$ penicillin/streptomycin (5000 units/mL of penicillin and $5000 \mu \mathrm{g} /$ $\mathrm{mL}$ of streptomycin, Thermo Fisher Scientific) with or without $30 \mathrm{ng} / \mathrm{mL}$ recombinant human IL-5 (R\&D Systems, Minneapolis, $\mathrm{MN}$ ) at $37^{\circ}$ in $5 \% \mathrm{CO}_{2}$ for $24 \mathrm{~h}$.

\section{Statistical analyses}

Mean \pm standard deviation ( $\bar{x} \pm S D)$, Pearson correlation coefficients (r), coefficients of variation, Student's t-test and analysis of covariance were calculated using Excel software (Microsoft, Redmond, WA).

\section{Results}

Between January 2014 and December 2018, we conducted 350 eosinophil purifications from 83 donors, yielding 341 successful purifications. Donors included 24 males and 59 females, ranging from 21-60 years of age. Among these 83 donors, 46 were allergic and 37 were non-allergic based on medical and medication history. Absolute eosinophil counts (AECs), calculated from the CBC results when additional blood was drawn for this purpose $(n=289)$, averaged $179 \pm 136$ eosinophils $/ \mu \mathrm{L}(\bar{x} \pm \mathrm{SD})$.

Eosinophil purity following immunomagnetic negative separation was consistently $>98 \%$, with contaminating cells being almost exclusively neutrophils (Fig. 1).

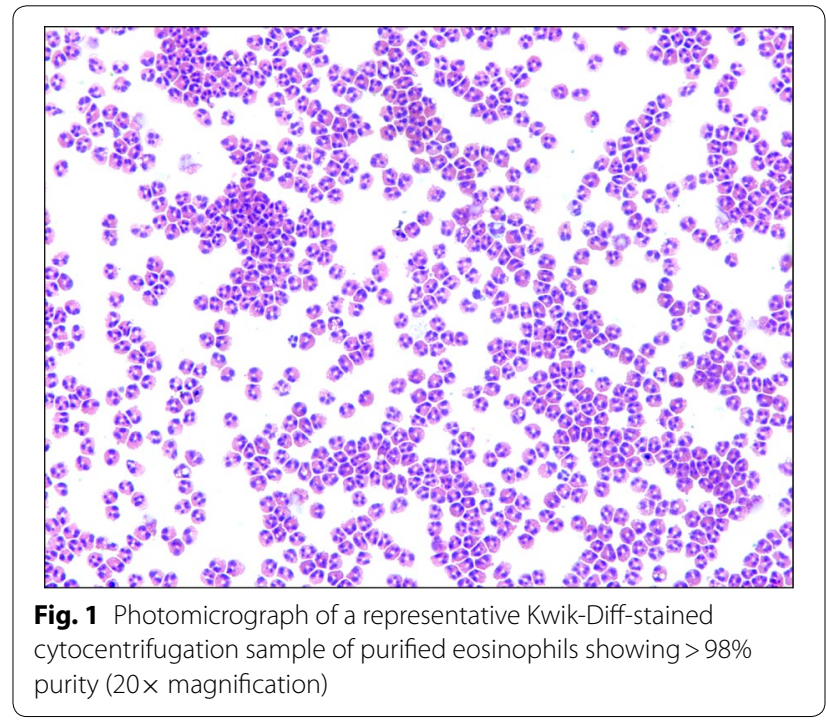


Eosinophil yields after 341 out of 350 successful purifications, calculated per $20 \mathrm{~mL}$ of blood drawn, ranged from 0.4 to 24.4 million cells $(\bar{x} \pm$ SD: $3.1 \pm 1.9$ million eosinophils, see Fig. 2a). Comparing AEC to actual yield, recovery was $87 \pm 29 \%(\bar{x} \pm S D)$ with a strong linear correlation between AEC and yield that did not differ statistically ( $p>0.5$ as assessed by analysis of covariance) between allergic and non-allergic donors (Pearson $r=0.9$ for allergics, $r=0.88$ for non-allergics and $r=0.897$ for all 289 donors) (Fig. 2b). To explore the reproducibility of yield, a subsequent analysis was limited to those donors whose blood was drawn $\geq 3$ times $(\mathrm{N}=35)$. With this approach, the average coefficient of variation for yield did not differ statistically between allergic $(\mathrm{N}=22)$ and nonallergic $(\mathrm{N}=13)$ donors $(27.5 \%$ versus $27.1 \%$, respectively, p $>0.5$ as assessed by Student's t-test) (Fig. 3), suggesting that there was no detectable impact of allergy history on eosinophil yield. Initial viability of isolated eosinophils was consistently $>95 \%$ and was $>90 \%$ after 24 h of culture with $30 \mathrm{ng} / \mathrm{mL}$ rhIL-5 versus $>80 \%$ without IL-5, and did not differ between allergic and non-allergic donors. Finally, regarding any incidental findings from the $\mathrm{CBC}$ results $(n=289)$, we did note the following: mild lymphopenia $(n=1)$, neutropenia $(n=4)$ or thrombocytopenia $(\mathrm{n}=2)$; anemia $(\mathrm{n}=4)$; slight ovalocytes $(\mathrm{n}=1)$, and large platelets $(n=1)$.

\section{Discussion}

To our knowledge, this report is the largest of its kind describing the results of commonly used methods for human eosinophil isolation from blood. Average
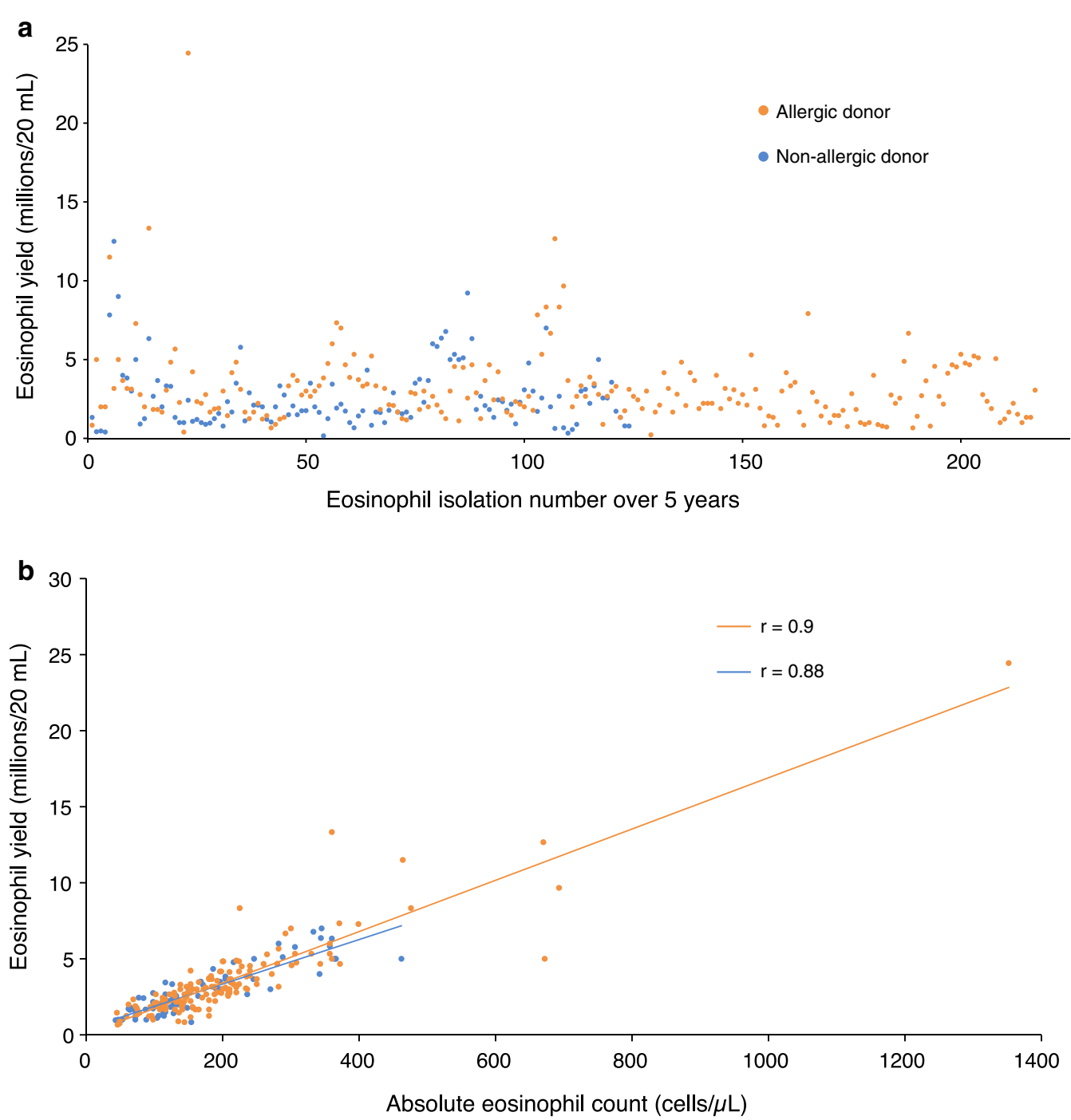

Fig. 2 Panel a. Eosinophil yield based on allergic status ( $n=341$ blood draws). Panel b. Correlations between eosinophil yield and absolute eosinophil count $(n=289)$ 


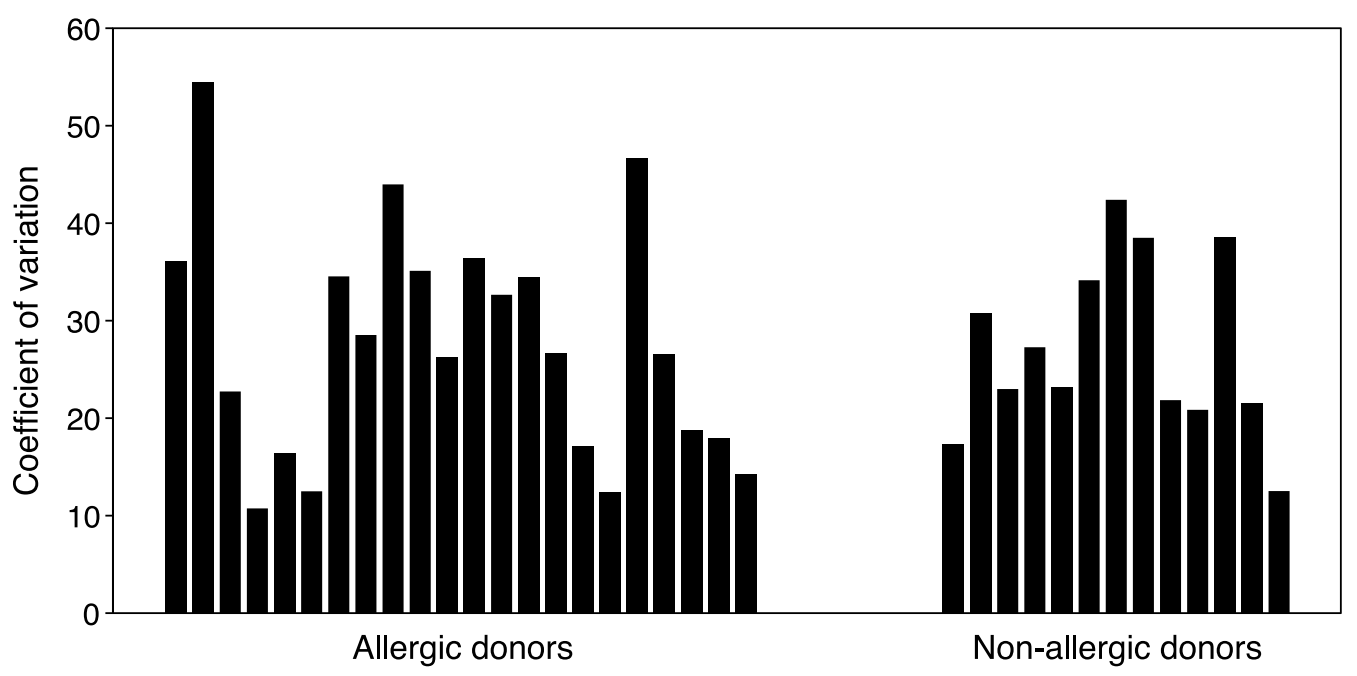

Fig. 3 Reproducibility of eosinophil yield for donors drawn $\geq 3$ times as assessed by calculations of coefficient of variation $(n=35)$

eosinophil yield per $20 \mathrm{~mL}$ of blood was $\approx 3$ million eosinophils with almost $90 \%$ recovery and consistently $>95 \%$ viability and purity. These were similar between allergic and non-allergic donors, as was their viability with or without IL-5 in culture for $24 \mathrm{~h}$. Yields per donor were fairly reproducible from donation to donation. Therefore, the described method for human eosinophil isolation from whole blood using density gradient centrifugation, $\mathrm{RBC}$ hypotonic lysis and immunomagnetic removal of neutrophils is a reliable, reproducible method for obtaining eosinophils at high yield, purity and viability.

\section{Limitations}

The main limitations of this study include the fact that all eosinophil isolations were performed at a single academic site by the same two people in the laboratory, with more than $80 \%$ being done by the first author. Other limitations are that the atopic status of each donor was not formally confirmed by traditional allergy testing. Most donors were not taking medications at the time of blood donation, so this should not be a limitation of this study.

\section{Abbreviations}

AEC: Absolute eosinophil count; CBC: Complete blood count with differential; DAPI: 4'6-diamidino-2-phenylindole dihydrochloride; RBC: Red blood cell.

\section{Acknowledgements}

The authors thank all 83 blood donors for participating in this project, most of whom donated on more than one occasion.

\section{Authors' contributions}

YC and SS did the eosinophil isolations; YC, SS, DJC and JAO performed the cell cultures and analyzed cell viability; YC and BSB did the data and statistical analyses, and generated the figures; BSB was responsible for consenting all blood donors, performing the phlebotomy and wrote the first draft of the manuscript, which was subsequently edited and approved by all authors. All authors read and approved the final manuscript.

Funding

This work was supported by National Institutes of Health NIH grants R01 Al072265, P01 HL107151 and U19 Al136443.

\section{Availability of data and materials}

The data supporting this publication is available at ImmPort (https://www. immport.org) under study accession SDY1627.

\section{Ethical approval and consent to participate}

Written informed consent for blood donation was obtained using an institutional review board-approved protocol at the Northwestern University Feinberg School of Medicine.

\section{Consent to publish}

Not applicable.

\section{Competing interests}

Dr. Bochner receives publication-related royalty payments from Elsevier and UpToDate. He serves as a consultant for Sanofi-Regeneron and GlaxoSmithKline. He receives remuneration for serving on the scientific advisory board of Allakos, Inc., and owns stock in Allakos. He is a coinventor on existing Siglec8-related patents and thus may be entitled to a share of royalties received by Johns Hopkins University during development and potential sales of such products. Dr. Bochner is also a cofounder of Allakos, which makes him subject to certain restrictions under University policy. The terms of this arrangement are being managed by the Johns Hopkins University and Northwestern University in accordance with their conflict of interest policies. All other authors declare no competing financial interests.

\section{Author details}

${ }_{1}^{1}$ Department of Medicine, Division of Allergy and Immunology, Northwestern University Feinberg School of Medicine, Chicago, IL 60611, USA. ${ }^{2}$ Division of Allergy and Immunology, Northwestern University Feinberg School of Medicine, 240 E. Huron St., Room M-306, Chicago, IL 60611, USA.

Received: 12 October 2019 Accepted: 1 April 2020

Published online: 10 April 2020 


\section{References}

1. Bochner BS. The eosinophil: for better or worse, in sickness and in health. Ann Allergy Asthma Immunol. 2018;121(2):150-5.

2. Khoury P, Bochner BS. Consultation for elevated blood eosinophils: clinical presentations, high value diagnostic tests, and treatment options. J Allergy Clin Immunol Pract. 2018;6(5):1446-53.

3. Guo C, Bochner BS. Workup for eosinophilia. Allergy Asthma Proc. 2019;40(6):429-32.

4. Tabatabaian F, Ledford DK, Casale TB. Biologic and new therapies in asthma. Immunol Allergy Clin North Am. 2017;37(2):329-43.

5. Kuang FL, Klion AD. Biologic agents for the treatment of hypereosinophilic syndromes. J Allergy Clin Immunol Pract. 2017;5(6):1502-9.

6. Legrand F, Klion AD. Biologic therapies targeting eosinophils: current status and future prospects. J Allergy Clin Immunol Pract. 2015;3(2):167-74.

7. Kuang FL, Legrand F, Makiya M, Ware J, Wetzler L, Brown T, Magee T, Piligian B, Yoon P, Ellis JH, et al. Benralizumab for PDGFRA-negative hypereosinophilic syndrome. N Engl J Med. 2019;380(14):1336-46.

8. Wechsler ME, Akuthota P, Jayne D, Khoury P, Klion A, Langford CA, Merkel PA, Moosig F, Specks U, Cid MC, et al. Mepolizumab or placebo for eosinophilic granulomatosis with polyangiitis. N Engl J Med. 2017;376(20):1921-32.

9. Panch SR, Bozik ME, Brown T, Makiya M, Prussin C, Archibald DG, Hebrank GT, Sullivan M, Sun X, Wetzler L, et al. Dexpramipexole as an oral steroid-sparing agent in hypereosinophilic syndromes. Blood. 2018;132(5):501-9.

10. O'Sullivan JA, Chang AT, Youngblood BA, Bochner BS. Eosinophil and mast cell Siglecs: from biology to drug target. J Leukoc Biol. 2020. https ://doi.org/10.1002/JLB.2MR0120-352RR.
11. Gartner I. Separation of human eosinophils in density gradients of polyvinylpyrrolidone-coated silica gel (Percoll). Immunology. 1980;40(1):133-6.

12. Blom M, Tool AT, Mul FP, Knol EF, Roos D, Verhoeven AJ. Eosinophils isolated with two different methods show different characteristics of activation. J Immunol Methods. 1995;178(2):183-93.

13. Fukuchi M, Ueki S, Saito H, Miyabe $Y$, Konno Y, Omokawa A, Saga T, Moritoki Y, Takeda M, Yamada T, et al. Comparison of CD16-negative selection vs. MACSxpress system for isolation of blood eosinophils. Allergol Int. 2019;68S:S11-3.

14. Son K, Mukherjee M, McIntyre BAS, Eguez JC, Radford K, LaVigne N, Ethier C, Davoine F, Janssen L, Lacy P, et al. Improved recovery of functionally active eosinophils and neutrophils using novel immunomagnetic technology. J Immunol Methods. 2017;449:44-55.

15. Wacht G, Poirot A, Charles AL, Radosavljevic M, Uring-Lambert B, de Blay F, Geny B, Bahram S, Barnig C. FACS-based isolation of human eosinophils allows purification of high quality RNA. J Immunol Methods. 2018;463:47-53.

16. Hansel TT, Pound JD, Pilling D, Kitas GD, Salmon M, Gentle TA, Lee SS, Thompson RA. Purification of human eosinophils by negative selection using immunomagnetic beads. J Immunol Methods. 1989;122:97-103.

\section{Publisher's Note}

Springer Nature remains neutral with regard to jurisdictional claims in published maps and institutional affiliations.
Ready to submit your research? Choose BMC and benefit from:

- fast, convenient online submission

- thorough peer review by experienced researchers in your field

- rapid publication on acceptance

- support for research data, including large and complex data types

- gold Open Access which fosters wider collaboration and increased citations

- maximum visibility for your research: over $100 \mathrm{M}$ website views per year

At BMC, research is always in progress.

Learn more biomedcentral.com/submissions 